Journal of ISMAC (2020)

Vol.02/ No. 01

Pages: $1-12$

http://irojournals.com/iroismac/

DOI: https://doi.org/10.36548/jismac.2020.1.001

\title{
EFFICIENT AND SECURE DATA UTILIZATION IN MOBILE EDGE COMPUTING BY DATA REPLICATION
}

\author{
Dr. N. Bhalaji, \\ Associate Professor, \\ Department of Information Technology, \\ SSN College of Engineering, \\ Old Mahabalipuram Rd, Kalavakkam, \\ Tamil Nadu - 603110. \\ Email id: bhalajin@ssn.edu.in
}

\begin{abstract}
The technological improvement at a rapid pace in the information and the communication fields has made the internet of things inevitable in our day today activities and takes a significant role in the every part of our regular schedule. The seamless communication through the internet of things is made possible, by connecting the tangible things around resulting in the numerous of advantages such as timely information delivery, servicing and monitoring. The inbuilt benefits of the IOT has made it more prominent among a wide range of application resulting in a huge data flow, though the congestion in the dataflow are managed using the cloud computing and the alternative sources such as the edge computing, the security of the data that are used are still under research. To manage the huge data flow and have secure data utilization in the internet of things, the paper has put forth the mobile edge computing integrated with the data duplication process taking into consideration the power utilization and the response time. The proposed method is simulated using the Network Simulator-2 and results obtained shows that the duplication process provides an enhancement in the bandwidth utilization along with the cut down in the power consumption and the response time.
\end{abstract}

Keywords: Data Replication (duplication), Cloud Computing, Edge Computing, Mobile Edge Computing, Internet of Things

\section{INTRODUCTION}

The internet of things is an interconnection of smart devices using heterogeneous networks and a wide paradigm for developing applications for the mobile/portable devices. IOT is a promising platform incorporating both the networking and the computing capabilities to make the tangible object communicate, with its states, to have a change in case if possible. To accomplish the tasks the internet of things includes embedded sensors, processors, actuators and the transceivers. The information from the physical world is conveyed using the sensor and the actuating devices. These devices which make the internet of things possible are equipped with the limited storage space, and require external storage for voluminous data. The cloud data centers with the massive data storage and high computing performance was usually preferred as external storage, but the over whelming data conveyance and the immediate access for the information's has made the cloud computing un-preferable. Especially for the resource constrained mobile devices that

ISSN: 2582-1369 (online) 
Journal of ISMAC (2020)

Vol.02/ No. 01

Pages: $1-12$

http://irojournals.com/iroismac/

DOI: https://doi.org/10.36548/jismac.2020.1.001

suffers to cope from the growing application devices. The emergence of edge computing behaves as a quiet good alternative for the cloud computing, decreasing the response time of the information's access.

The edge computing is clubbed with the mobile computing to make conceivable the application that demands high bandwidth utilization and with the low latency, this termed as the mobile edge computing, the mobile edge computing extends the services of the cloud paradigm to its edge to the mobile base stations. This technology seems to be promising as the as the edge technology is applied to the mobile devices affording the information transfer and the service options at the outskirts of its users and the seamless integration of numerous applications such as the subscribers, providers, vendors and organizations [7].

Some of the related terms that are similar to the mobile edge computing are (i) mobile cloud computing, that actually clubs the advantages of the mobile computing with the cloud paradigm and the internet of mobile, (ii) local cloud, that are particularly meant for a single organization, (iii) cloudlet, that are data centers of small size located at the few distance away from the mobile devices and (iv) Fog computing, the fog computing and the MEC are interchangeably used the intelligence of the fog and the MEC differs as the MEC's communication competence and the intelligence are driven by the RAN, whereas FOG depends on the LAN and also faces some issues related to the dependency in the wireless connections. The architectural depiction of the mobile edge cloud is presented below in the figure. 1

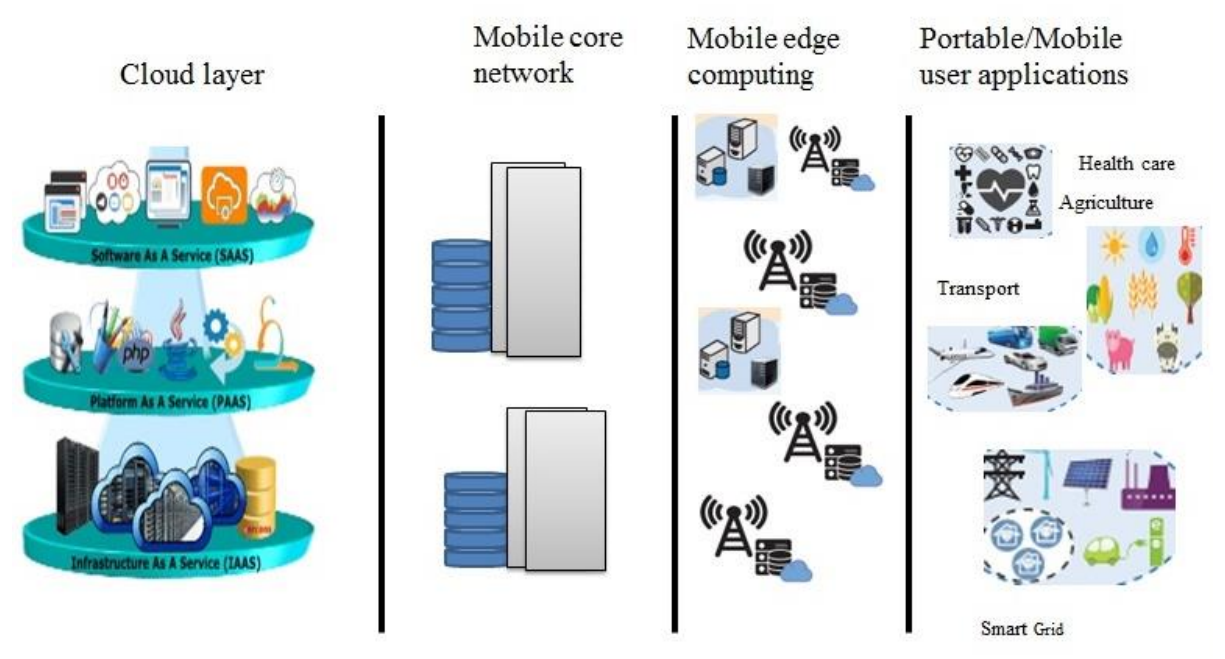

Figure.1 Architecture of Mobile Edge Computing

ISSN: 2582-1369 (online) 
Journal of ISMAC (2020)

Vol.02/ No. 01

Pages: $1-12$

http://irojournals.com/iroismac/

DOI: https://doi.org/10.36548/jismac.2020.1.001

It is a three layer architecture where the MEC is place between the cloud and the mobile devices. According the ETSI white papers the MEC has the following attributes, the mobile edge computing are less vulnerable due to their segregated performance, beneficial for compute hungry devices as they are placed at proximity, provides high bandwidth utilization and low latency, aware of location and provides network context information.

Due to the numerous benefits provided by the MEC, the paper clubs the mobile edge computing for improving the data utilization in IOT to elude the data losses securing the information as well as cut down the cost of storage and the delay admitted in response using the data duplication.

The rest of the paper is organized with the part 2. Providing the details of the work referred to develop the proposed system, part 3 with the proposed data duplication, part 4 with the result analysis and part 5 with the conclusion.

\section{RELATED WORKS}

The duplication of the data sets are always considered to be more crucial for the purpose of speedy access, bandwidth consumption and the waiting time the data replication in the cloud computing was considered to be time as well as cost consuming so many found that the fog computing would be an effective alternative to hold the replicated data's as it consumes less time, power and cost considered to the cloud so Bestak, et al [1] in his paper proposes an fog service for the big data analytics of the health care services to reduce the time required in accessing the sensitive data's, as majority of the data flow is encountered from the internet of things Smys et al [2] presents the report about the protocols involved architecture and the applications of the internet of things and the Jennifer S. Raj.et al [3]presents the "A Stochastic Mobile Data Traffic Model For Vehicular Ad Hoc Networks."

Though the fog computing reduce the time, power and cost the still were unable to provide a massive storage support as the they did not have sufficient space as the cloud computing, so the author Karunakaran, $\mathrm{V}$ et al [4] put forth the an improved task scheduling method to reduce the time consumption in the completion of the work and the author Bhalaji, N.et al [5] put forth the techniques to reduce the delay in the scheduling of the tasks for the cloud environment that is heterogeneous. They didn't concentrate in duplication but in the scheduling to achieve the reduced time utilization, the duplication process is classified as the decision on duplication and the placement of the duplicated data's the figure .2 presents the process and the types of the duplication.

ISSN: 2582-1369 (online) 
Journal of ISMAC (2020)

Vol.02/ No. 01

Pages: $1-12$

http://irojournals.com/iroismac/

DOI: https://doi.org/10.36548/jismac.2020.1.001

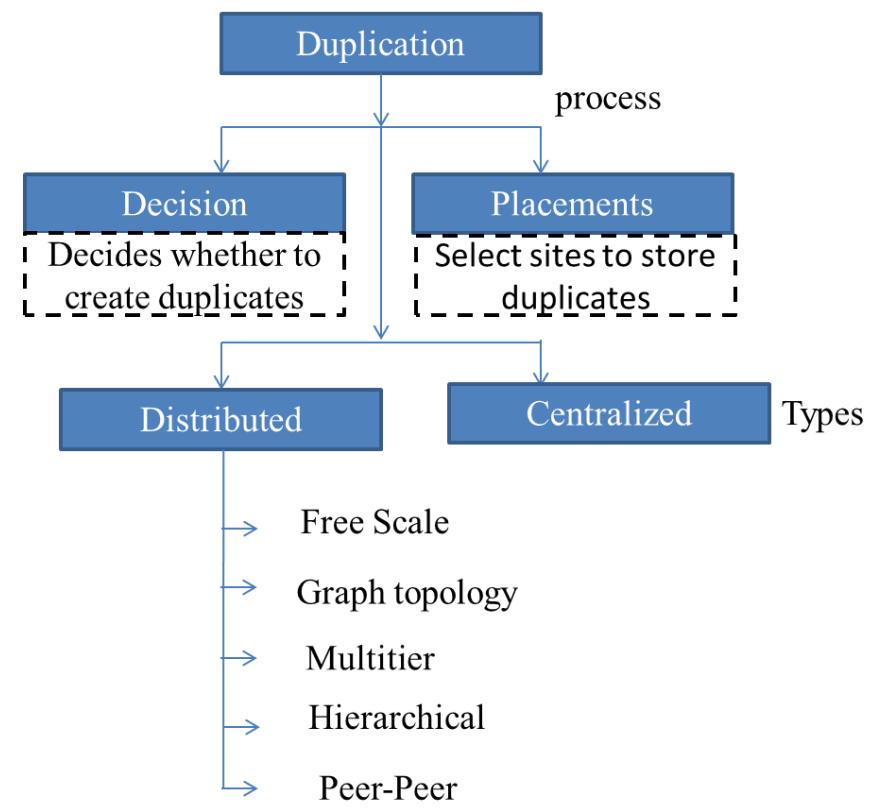

Figure .2 Process and Types of Duplication

Nowadays as the mobile edge computing is considered as the good alternative for the Cloud and the Fog the author Abbas, et al [6] provides the survey on the MEC detailing the limitations of it similar terms and its architecture and Harikumar, $\mathrm{R}$ et al [7] provides the "Ad hoc node connectivity improvement analysisthrough mesh clients." and Roman, Rodrigo et al [8] also present the survey of the MEC as an matching service to the fog. Yu, Wei et al [9] "puts forth the essentials of the edge computing for the internet of things as a survey"

The author Zhang, et al [10] highlights in his paper how the mobile edge computing can be used in making the internet of things green with the low latency. Tiwary et al [11] puts forth the game model to reduce the latency for cloudlets in the MEC by providing an optimal offloading decision. The security concerns being a major problem for the information's gathered through the internet of things the author S. Smys et al [12] proposes the "intelligent security frame work for the internet of things through the cryptographic techniques". Sharnya, P et al [13] proffers "Self organizing wireless mesh network." Sultana, et al [14] "Efficient resource allocation in device-to-device communication using cognitive radio technology" and Shi, Yong et al [15] proposes the load balancing task scheduling through the cloud by the load balancing so as to reduce the time, consumption.

ISSN: 2582-1369 (online) 
Journal of ISMAC (2020)

Vol.02/ No. 01

Pages: $1-12$

http://irojournals.com/iroismac/

DOI: https://doi.org/10.36548/jismac.2020.1.001

The above methods do not consider the costs on the basis of the duplication creation and placements and also does not address the utilization and the security demands of the data gathered from the internet of things, so the proposed method puts forth the proper placement of the data replications so as to reduce the cost, time, power utilization enhancing the data utilization using the random replication algorithm[16] and assures security for the data using the advanced cryptographic techniques [17].

\section{PROPOSED WORK}

The duplication [18] of the data's is really in effect as it improves the data accessibility reducing the latency as well as the availability of the data, the proposed system of data duplication [19] through the mobile edge computing concentrates on system performance, data utilization and also on the security of the data, as the internet service today finds hard in managing the traffic of data causing congestions resulting in prolonged delays. The replication [20] process is usually duplicating the original data and placing it at proximity to the users so that they can utilize the data any time without delay, this was previously achieved using the cloud computing, but the latency and cost problems strived for a better results, and paved way for a closer storage to the user rather than the remote storage and the mobile edge computing that brought the services of the cloud to the user edge was conceived. The proposed method in the paper further strives to reduce the latency, improving the data utilization along with the security through the data duplication (replication) in the mobile edge computing. The proposed method utilizes the random replication algorithm [16] and the blowfish techniques [17] to duplicate the data and provide security for the data respectively, the figure. 3 below shows the overview of the proposed method.

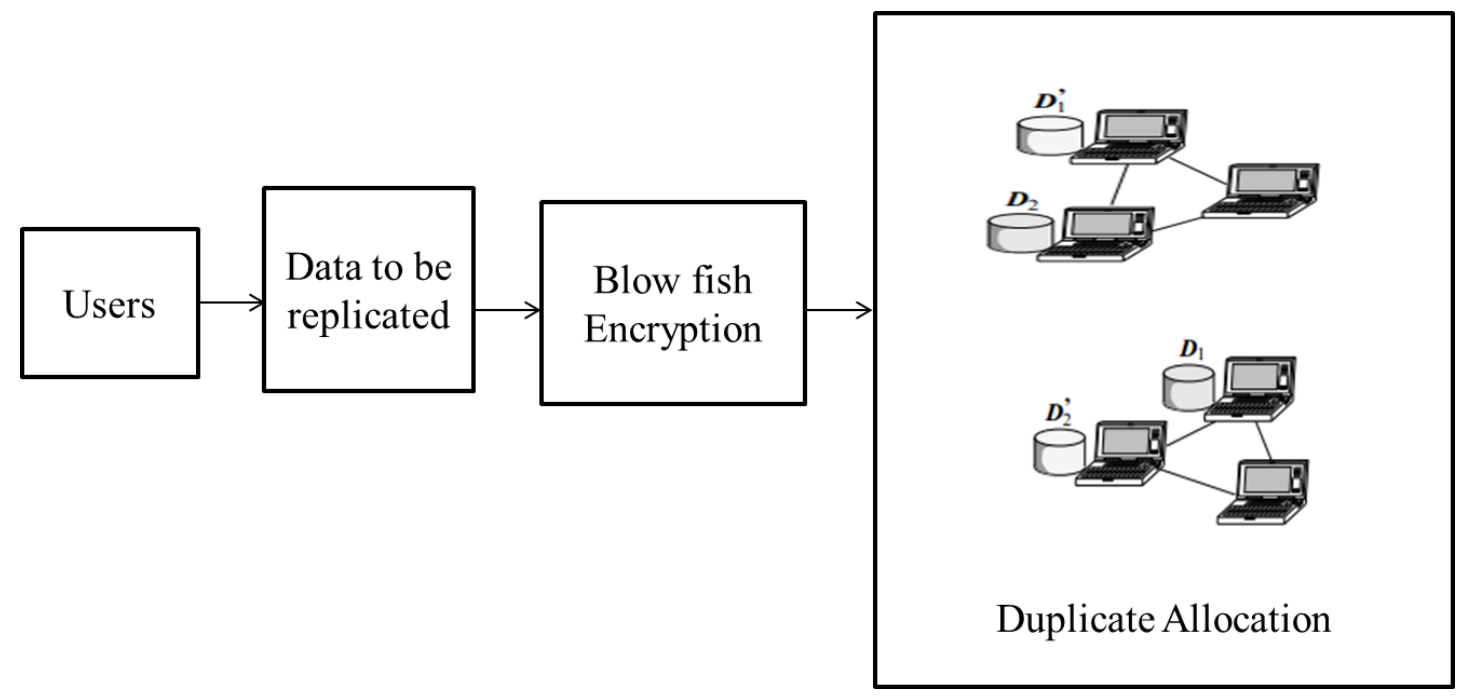

Figure.3 Overview of Proposed Method

ISSN: 2582-1369 (online) 
Journal of ISMAC (2020)

Vol.02/ No. 01

Pages: $1-12$

http://irojournals.com/iroismac/

DOI: https://doi.org/10.36548/jismac.2020.1.001

\subsection{RANDOMIZED DUPLICATION}

The random replication method is simple duplication procedure that relies on the principle of the simple replication algorithm that utilizes duplication creation in nodes to initialize the hop counter to zero. Later the mobile nodes would start diffusing the duplicate messages to its neighbors, each time the duplication message is send to the neighboring nodes the counter is incremented and once again initialized to zero if the replica exists on the node visited. This process is followed by the random duplication (RD) methods for the distribution of duplicates, The RD prioritizes its duplicates based on how frequently the data is retrieved $\left(R_{\text {freq }}\right)$ and sends the rarely accessed data's to the remote locations for storage and also eludes the redundancies. The flow chart below in figure. 4 shows the Random duplication procedure.

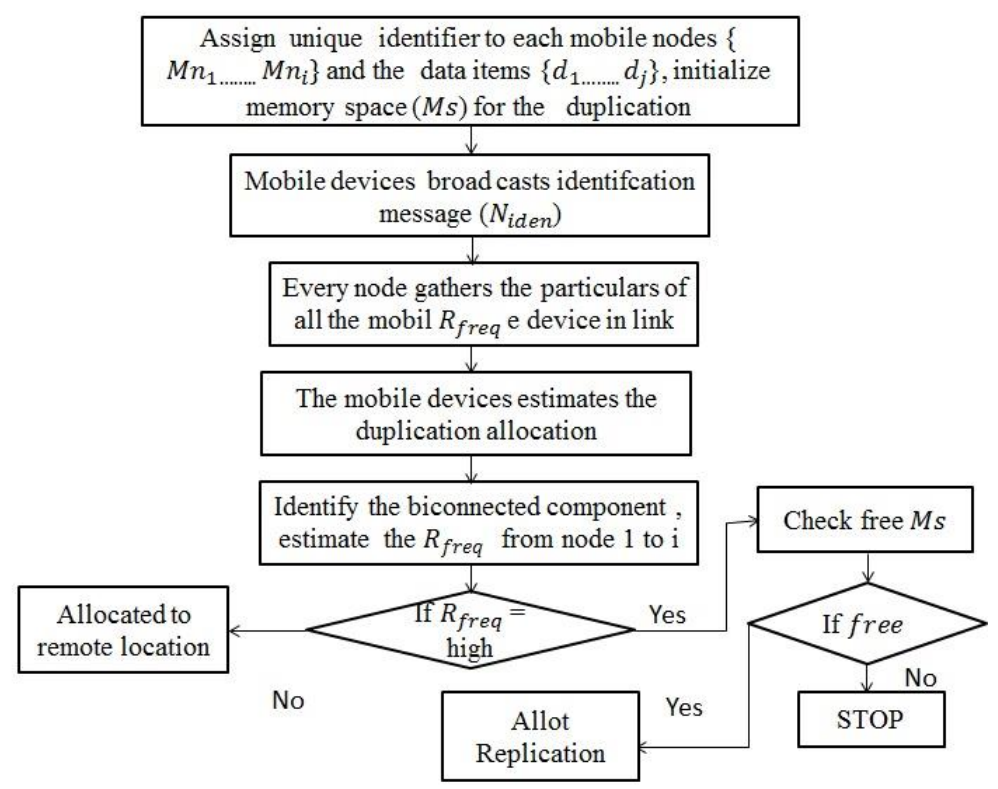

Figure.4 Randomized Duplication Allocation

The dynamic connectivity based grouping [21] is followed in the randomized allocation of the duplications, the optimality in the allocation is determined analytically considering the parameters, $R_{\text {freq }}$, participation probability of the mobile devices $\left(\mathrm{Par}_{\text {prob }}\right)$, disappearance probability ( $\left.\mathrm{Di} S_{P R o b}\right)$, probability that the biconnected nodes will be connected only through either of one links whichever is first, the allocation of the replications $\left(\operatorname{Rep}_{\text {alloc }}\right)$ based on the following equation (1)

ISSN: 2582-1369 (online) 
Journal of ISMAC (2020)

Vol.02/ No. 01

Pages: $1-12$

http://irojournals.com/iroismac/

DOI: https://doi.org/10.36548/jismac.2020.1.001

$$
\operatorname{Rep}_{\text {alloc }}=\left\{\frac{d 1 !}{d 1-M S}\right\}^{i 1}
$$

Where ' $\mathrm{i}$ ' is the number of mobile devices, and the ' $d$ ' is the data items and the 'Ms' is the memory space, and the density of the network $\left(N_{d}\right)$ is estimated using the ' $M n^{\prime}$, the area of coverage $\left(A v g_{C A}\right)$ and the Total area $(T A)$.

$$
N_{d}=\frac{M n \times A v g_{C A}}{T A}
$$

\subsection{BLOWFISH ENCRYPTION/DECRYPTION}

The symmetric block encryption / decryption methodology is employed for the data to protect the data from the attackers at the mobile edge; the personal data duplicated in multiple nodes are sometimes liable of be hacked through the attacker mobile devices in the network. So to avoid such problems the as mentioned in the architectural over view in the figure .3 the data are encrypted before allocation and then duplicated, the identification allotted to the each data enables the replication identification to elude redundancies at ease. The blow fish is preferred in the proposed model as it does a faster encryption as well as decryption; utilizing very less memory $(<5 \mathrm{~K})$, additionally the blowfish uses XOR and look up table that has 32-bit operands. The figure .5 shows the cryptographic methodology followed in the blowfish.
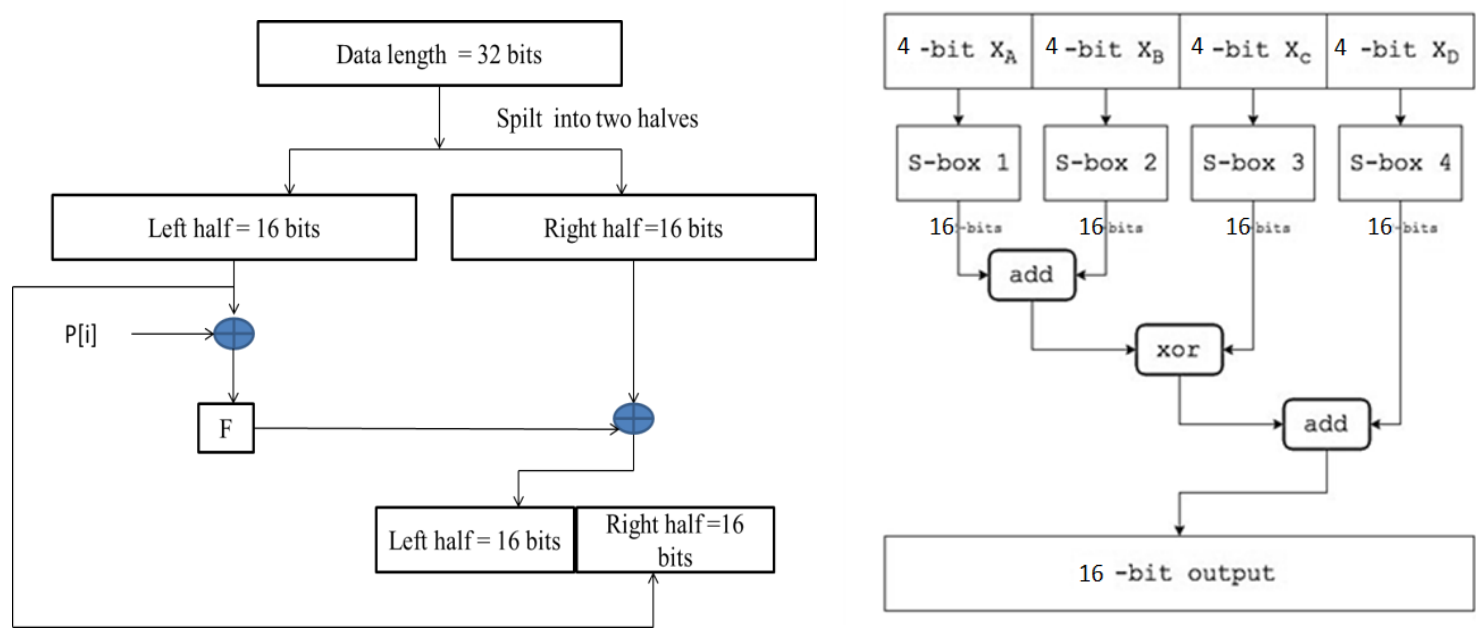

Figure.5 Blowfish Encryption (Left) and Flow diagram of 'F' (Right)

ISSN: 2582-1369 (online) 
Journal of ISMAC (2020)

Vol.02/ No. 01

Pages: $1-12$

http://irojournals.com/iroismac/

DOI: https://doi.org/10.36548/jismac.2020.1.001

Where $\mathrm{P}[\mathrm{i}]$ is the sub-keys ranging from $\{\mathrm{p}[0]-\mathrm{p}[7]\}$. The decryption follows just the reverse process, the proposed methodology engages the blowfish to encrypt the data and then allots the identification to the each data and replicates the data and allocates according to the equation (1) as shown in the figure.3, to avoid overhead the and congestion the mobile nodes exchange the information's and performs duplication reallocation following the same methodology.

\section{RESULT EVALUATION}

The proposed model is evaluated using the network simulator- II for 50 to 100 mobile edge nodes, with 100 to 200 data's to be replicated, with the capacity of $10-50$, the proposed method is simulated along with the other conventional methods that employ replication without security and the method that does not employ the duplication. The latency in the response, the data utilization percentage and the security percentage for the above methods were evaluated and compared. The table.1 below shows the time taken for the encryption, decryption and the memory used for different data set.

\begin{tabular}{|c|c|c|c|c|}
\hline $\begin{array}{c}\text { File Size } \\
(\mathrm{KB})\end{array}$ & $\begin{array}{c}\text { Encryption } \\
\text { Time (ms) }\end{array}$ & $\begin{array}{c}\text { Decryption } \\
\text { Time (ms) }\end{array}$ & $\begin{array}{c}\text { Memory } \\
\text { Used (KB) }\end{array}$ & $\begin{array}{c}\text { Entropy per } \\
\text { byte }\end{array}$ \\
\hline 50 & 200 & 50 & 2 & 1.2 \\
\hline 100 & 250 & 150 & 2.5 & 2.4 \\
\hline 1024 & 300 & 300 & 2.8 & 2.7 \\
\hline 2048 & 400 & 350 & 3 & 3.09 \\
\hline
\end{tabular}

Table.1 Blow Fish Encryption/Decryption

The figure. 6 is the result observed for the latency in the response, for the three methods, proposed, conventional with replication, without security (CWR) and conventional without replication and security (CWRS). The results obtained shows that the latency of the proposed method is slightly higher than the conventional with replication and without security, for lesser number of mobile devices and becomes stable as the nodes increase, the slight increase is experienced due to the use cryptographic methods in the data to ensure the security of the data and very less compared to the conventional method that is without duplication and security.

ISSN: 2582-1369 (online) 
Journal of ISMAC (2020)

Vol.02/ No. 01

Pages: 1-12

http://irojournals.com/iroismac/

DOI: https://doi.org/10.36548/jismac.2020.1.001

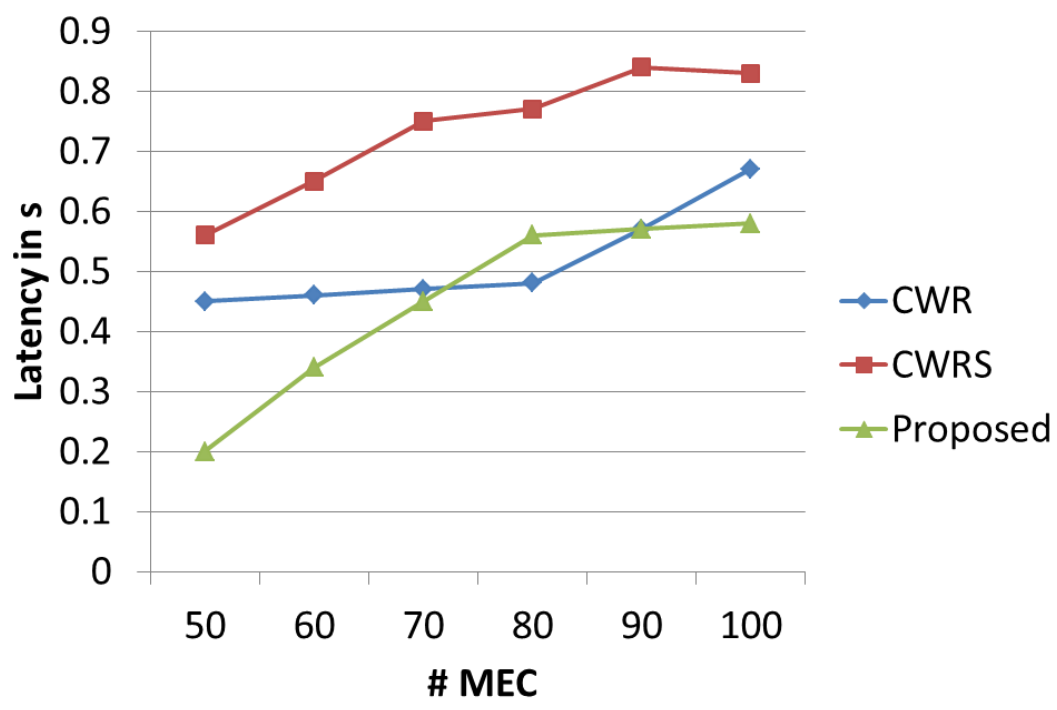

Figure.6 Latency observed

The figure. 7 shows the data utilization percentage and the security percentage of the proposed, conventional with replication, without security and conventional without replication and security. The results obtained shows the proposed method has attained a $25 \%$ and $70 \%$ higher data utilization and security compared to the conventional with replication and without security, and $50 \%$ and $89 \%$ higher data utilization and security compared to the conventional method that is without duplication and security.

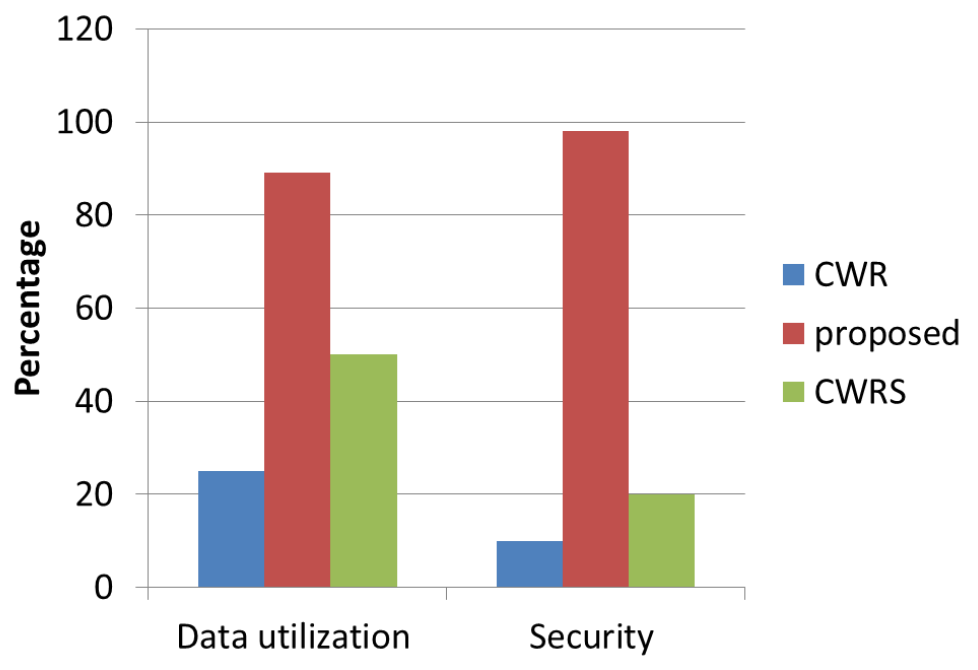

ISSN: 2582-1369 (online) 
Journal of ISMAC (2020)

Vol.02/ No. 01

Pages: $1-12$

http://irojournals.com/iroismac/

DOI: https://doi.org/10.36548/jismac.2020.1.001

Figure.7 Data Utilization and Security

The table. 2 below provides the cost optimization of the proposed method, conventional with replication, without security and conventional without replication and security, for 100-200 of replicated data's

\begin{tabular}{|c|c|c|c|c|}
\hline $\begin{array}{c}\text { Number of } \\
\text { Replicated } \\
\text { Data }\end{array}$ & $\begin{array}{c}\text { Transfer } \\
\text { Cost \% }\end{array}$ & $\begin{array}{c}\text { Storage } \\
\text { Cost } \%\end{array}$ & Total Cost \% & $\begin{array}{c}\text { Bandwidth } \\
\text { Savings \% }\end{array}$ \\
\hline 100 & 5 & 5.5 & 5 & 78 \\
\hline 110 & 7 & 9 & 10 & 79 \\
\hline 120 & 7.5 & 9.2 & 10 & 79.5 \\
\hline 140 & 8 & 11 & 12 & 84 \\
\hline 180 & 8.2 & 11.4 & 13 & 85.3 \\
\hline 200 & 9 & 12 & 12 & 86 \\
\hline
\end{tabular}

Table. 2 Cost Optimization

\section{CONCLUSION}

The proposed method using the randomized duplication and the security enhances the data utilization and the security provisions in mobile edge computing for the internet of things data, the blow fish encryption/decryption with the fastest encryption and decryption and the less memory usage ensure the security of the data by eluding the information's even from the guessing attacks. The results obtained shows that the proposed method has the highest percentage of improvement in the performance compared to the conventional methods. 
Journal of ISMAC (2020)

Vol.02/ No. 01

Pages: $1-12$

http://irojournals.com/iroismac/

DOI: https://doi.org/10.36548/jismac.2020.1.001

\section{References}

[1] Bestak, Robert, and S. Smys. "BIG DATA ANALYTICS FOR SMART CLOUD-FOG BASED APPLICATIONS." Journal of trends in Computer Science and Smart technology (TCSST) 1, no. 02 (2019): 74-83.

[2] Kumar, R. Praveen, and S. Smys. "A novel report on architecture, protocols and applications in Internet of Things (IoT)." In 2018 2nd International Conference on Inventive Systems and control (ICISC), pp. 1156-1161. IEEE, 2018.

[3] Smys, S., and Jennifer S. Raj. "A STOCHASTIC MOBILE DATA TRAFFIC MODEL FOR VEHICULAR AD HOC NETWORKS." Journal of Ubiquitous Computing and Communication Technologies (UCCT) 1, no. 01 (2019): 55-63.

[4] Karunakaran, V. "A STOCHASTIC DEVELOPMENT OF CLOUD COMPUTING BASED TASK SCHEDULING ALGORITHM." Journal of Soft Computing Paradigm (JSCP) 1, no. 01 (2019): 41-48.

[5] Bhalaji, N. "DELAY DIMINISHED EFFICIENT TASK SCHEDULING AND ALLOCATION FOR HETEROGENEOUS CLOUD ENVIRONMENT." Journal of trends in Computer Science and Smart technology (TCSST) 1, no. 01 (2019): 51-62.

[6] Abbas, Nasir, Yan Zhang, Amir Taherkordi, and Tor Skeie. "Mobile edge computing: A survey." IEEE Internet of Things Journal 5, no. 1 (2017): 450-465.

[7] Harikumar, R., and Jennifer S. Raj. "Ad hoc node connectivity improvement analysis-Why not through mesh clients?." Computers \& Electrical Engineering 40, no. 2 (2014): 473-483.

[8] Roman, Rodrigo, Javier Lopez, and Masahiro Mambo. "Mobile edge computing, fog et al.: A survey and analysis of security threats and challenges." Future Generation Computer Systems 78 (2018): 680-698.

[9] Yu, Wei, Fan Liang, Xiaofei He, William Grant Hatcher, Chao Lu, Jie Lin, and Xinyu Yang. "A survey on the edge computing for the Internet of Things." IEEE access 6 (2017): 69006919.

[10] Zhang, Ke, Supeng Leng, Yejun He, Sabita Maharjan, and Yan Zhang. "Mobile edge computing and networking for green and low-latency Internet of Things." IEEE Communications Magazine 56, no. 5 (2018): 39-45.

[11] Tiwary, Mayank, Deepak Puthal, Kshira Sagar Sahoo, Bibhudatta Sahoo, and Laurence T. Yang. "Response time optimization for cloudlets in Mobile Edge Computing." Journal of Parallel and Distributed Computing 119 (2018): 81-91.

[12] Sridhar, S., and S. Smys. "Intelligent security framework for iot devices cryptography based end-to-end security architecture." In 2017 International Conference on Inventive Systems and Control (ICISC), pp. 1-5. IEEE, 2017.

[13] Sharnya, P., and Jennifer S. Raj. "Self organizing wireless mesh network." International Journal of Innovation and Applied Studies 3, no. 2 (2013): 486-492. 
Journal of ISMAC (2020)

Vol.02/ No. 01

Pages: $1-12$

http://irojournals.com/iroismac/

DOI: https://doi.org/10.36548/jismac.2020.1.001

[14] Sultana, Ajmery, Lian Zhao, and Xavier Fernando. "Efficient resource allocation in device-todevice communication using cognitive radio technology." IEEE Transactions on Vehicular Technology 66, no. 11 (2017): 10024-10034.

[15] Shi, Yong, and Kai Qian. "LBMM: a load balancing based task scheduling algorithm for cloud." In Future of Information and Communication Conference, pp. 706-712. Springer, Cham, 2019.

[16] Fan, Xiying, Chuanhe Huang, Junyu Zhu, and Bin Fu. "R-DRA: a replication-based distributed randomized algorithm for data dissemination in connected vehicular networks." Wireless Networks 25, no. 7 (2019): 3767-3782.

[17] Wahid, Mohammed Nazeh Abdul, Abdulrahman Ali, Babak Esparham, and Mohamed Marwan. "A Comparison of Cryptographic Algorithms: DES, 3DES, AES, RSA and Blowfish for Guessing Attacks Prevention." Journal Computer Science Applications and Information Technology 3 (2018): 1-7.

[18] Wu, Xiuguo. "Combination Replicas Placements Strategy for Data sets from Cost-effective View in the Cloud." International Journal of Computational Intelligence Systems 10, no. 1 (2017): 521-539.

[19] Mazumdar, Somnath, Daniel Seybold, Kyriakos Kritikos, and Yiannis Verginadis. "A survey on data storage and placement methodologies for cloud-big data ecosystem." Journal of Big Data 6, no. 1 (2019): 15.

[20] Tos, Uras. "Data replication in large-scale data management systems." PhD diss., 2017.

[21] Hara, Takahiro. "Effective replica allocation in ad hoc networks for improving data accessibility." In Proceedings IEEE INFOCOM 2001. Conference on Computer Communications. Twentieth Annual Joint Conference of the IEEE Computer and Communications Society (Cat. No. 01CH37213), vol. 3, pp. 1568-1576. IEEE, 2001.

ISSN: 2582-1369 (online) 